\title{
Second variation of one-sided complete minimal surfaces
}

\author{
Francisco Urbano
}

\begin{abstract}
The stability and the index of complete one-sided minimal surfaces of certain 3-dimensional Riemannian manifolds with positive scalar curvature are studied.
\end{abstract}

\section{Introduction}

The study of the second variation of the volume of minimal submanifolds in Riemannian manifolds is a classical problem in differential geometry. In fact, the second variation operator (the Jacobi operator) carries information about the stability properties of the submanifold when it is considered as a stationary point for the volume functional. An important particular case in this setting is when the submanifold is a hypersurface. In this case, the rank of the normal bundle is one, and we can consider two different situations: the hypersurface is two-sided, i.e., the normal bundle is trivial, or the hypersurface is one-sided, i.e., the normal bundle is nontrivial. In the first case, we can define a global unit normal vector field which trivializes the normal bundle. Then the Jacobi operator, which acts on the sections of the normal bundle, becomes a Schrödinger operator acting on functions. This case, perhaps the easiest one, has been studied by many people (see [2], [1], [4], [5], $[6],[11],[16]$ and the references therein). For one-sided minimal hypersurfaces and for minimal submanifolds with high codimension, only a few particular situations have been considered (see [7], [8], [9], [10], [12], [13], [14], [15] and the references therein).

In the present work, we are interested in the second variation of complete minimal surfaces of Riemannian 3-manifolds. Many interesting results are well known for compact surfaces. When $M$ is the 3 -sphere $\mathbb{S}^{3}$, Simons [14] proved that the index of any compact minimal surface of $\mathbb{S}^{3}$ is at least one (in particular there are no stable ones) and the totally geodesic equators are the only ones with index one. Later, Urbano [16] classified the orientable compact minimal surfaces of $\mathbb{S}^{3}$ with index less than six, proving that the surface must be either an equator or the Clifford

Mathematics Subject Classification (2010): 53C40, 53C42.

Keywords: Stability, minimal surfaces, index. 
torus which has index five. When $M$ is the real projective space $\mathbb{R P}^{3}$, Onhita [9] proved that its only stable compact minimal surface is the totally geodesic real projective plane, and later, Do Carmo, Ritoré and Ros [2] characterized the totally geodesic two-sphere and the Clifford torus as the only orientable two-sided compact minimal surfaces of $\mathbb{R P}^{3}$ with index one.

For complete two-sided minimal surfaces in 3-manifolds, the starting point was the characterization of the plane as the only complete stable two-sided minimal surface in the Euclidean space $\mathbb{R}^{3}$ (see [5], [1], [11]). Later, López-Ros [6] proved that the catenoid and Enneper's surface are the only two-sided complete minimal surfaces of $\mathbb{R}^{3}$ with index one. Fischer-Colbrie and Schoen [5], [4] studied in depth the second variation of complete two-sided minimal surfaces of 3-dimensional Riemannian manifolds with nonnegative scalar curvature, analyzing, on any complete Riemannian surface, the index of the Schrödinger operator $L=\Delta-K+q$, where $\Delta$ is the Laplacian, $K$ is the Gauss curvature of the surface and $q$ is a nonnegative function. In fact, the Jacobi operator of such minimal surfaces can be written in the above form. Also, in this setting, it is interesting to remark [5], [6] that a complete finite index two-sided minimal surface of a 3-dimensional Riemannian manifold with scalar curvature $\rho \geq \delta>0$ must be compact.

For complete one-sided minimal surfaces in 3-manifolds, the theory of FischerColbrie and Schoen cannot be applied, and only some particular situations have been studied. Perhaps, the most interesting paper in this direction is the paper [12] of Ros, where he uses new ideas and proves, among other things, that there are no complete stable one-sided minimal surfaces in $\mathbb{R}^{3}$. A partial case of this was proved by Ross in [13].

In this paper, we study the stability and the index of complete minimal surfaces of $\mathbb{S}^{3}, \mathbb{S}^{2} \times \mathbb{R}$, and some of their Riemannian quotients like $\mathbb{R P}^{3}, \mathbb{R P}^{2} \times \mathbb{R}, \mathbb{S}^{2} \times \mathbb{S}^{1}$ and $\mathbb{R P}^{2} \times \mathbb{S}^{1}$. The main results in the paper are summarized in the following:

The index of any complete and non compact minimal surface of $\mathbb{S}^{3}$, $\mathbb{R} \mathbb{P}^{3}, \mathbb{S}^{2} \times \mathbb{R}$ or $\mathbb{S}^{2} \times \mathbb{S}^{1}$ is infinite.

The totally geodesic embedding $\mathbb{R P}^{1} \times \mathbb{R} \subset \mathbb{R P}^{2} \times \mathbb{R}$ is the only stable orientable complete and non compact minimal surface of $\mathbb{R P}^{2} \times \mathbb{R}$.

The totally geodesic embedding $\mathbb{S}^{1} \times \mathbb{S}^{1} \subset \mathbb{S}^{2} \times \mathbb{S}^{1}$ is the only compact orientable minimal surface of $\mathbb{S}^{2} \times \mathbb{S}^{1}$ with index one.

We remark that, when the surface is two-sided, the first result follows from FischerColbrie and Schoen theory. Also, $\mathbb{R P}^{1} \times \mathbb{R}$ is an orientable one-sided minimal surface in $\mathbb{R P}^{2} \times \mathbb{R}$.

The author would like to thank A. Ros for his valuable comments on the paper.

\section{Harmonic vector fields on surfaces}

As in the proofs of some results we will use harmonic vector fields as test functions, in this section we recall some properties about harmonic vector fields, which will be used throughout the paper. 
Given an orientable Riemannian surface $\Sigma$, a vector field $X$ on $\Sigma$ is harmonic if the associated 1-form $\omega_{X}$ is harmonic, i.e., $\omega_{X}$ is closed and coclosed. This means that $\operatorname{div}(X)=0$ and $\nabla X$ is a symmetric tensor, where div is the divergence on $\Sigma$ and $\nabla$ is the Levi-Civita connection on $\Sigma$.

If $\Delta^{\Sigma}$ is the Laplacian on $\Sigma$ acting on vector fields and $X$ is a harmonic vector field, it is easy to check that

$$
\Delta^{\Sigma} X=K X
$$

where $K$ is the Gauss curvature of $\Sigma$. Also, if $J$ is the complex structure on the Riemann surface $\Sigma$, then $X$ is harmonic if and only if $J X$ is harmonic. Let $H(\Sigma)$ be the space of square integrable harmonic vector fields on $\Sigma$. Then, if $\Sigma$ is compact of genus $g$, we have that $\operatorname{dim} H(\Sigma)=2 g$. If $\Sigma$ is non compact, then $\operatorname{dim} H(\Sigma) \geq$ 2genus $(\Sigma)$, including the case where the genus of $\Sigma$ is infinite (see [3], p. 42).

If $\Sigma$ is a non orientable Riemannian surface and $(\tilde{\Sigma}, \tau)$ is its two-fold oriented covering, where $\tau$ is the change of sheet, then any harmonic vector field $X$ on $\tilde{\Sigma}$ decomposes as $X=X^{+}+X^{-}$, where $X^{+}$and $X^{-}$are harmonic vector fields satisfying $\tau_{*} X^{+}=X^{+}, \tau_{*} X^{-}=-X^{-}$and $J X^{-}=X^{+}$. In this case, $H(\tilde{\Sigma})=$ $H^{+}(\tilde{\Sigma}) \oplus H^{-}(\tilde{\Sigma})$, where $H^{ \pm}(\tilde{\Sigma})=\left\{X \in H(\tilde{\Sigma}) \mid \tau_{*} X= \pm X\right\}$ and $J: H^{+}(\tilde{\Sigma}) \rightarrow$ $H^{-}(\tilde{\Sigma})$ is an isomorphism.

It is interesting to note an easy property which will be used in the paper. Given a $k$-dimensional subspace $V$ of $H(\Sigma)$, there exists an integrable function $h$ on $\Sigma$ such that $|X|^{2} \leq h$ for any $X \in V$ with $\int_{\Sigma}|X|^{2}=1$. In fact if $\left\{V_{1}, \ldots, V_{k}\right\}$ is a $L^{2}$-orthonormal basis of $V$, then $X=\sum_{i=1}^{k} \lambda_{i} V_{i}$ with $\sum \lambda_{i}^{2}=1$. Now it is clear that $h$ can be taken as the integrable function $k^{2} \max \left\{\left\langle V_{i}, V_{j}\right\rangle, 1 \leq i, j \leq k\right\}$.

\section{Jacobi operator}

Let $\Phi: \Sigma \rightarrow\left(M^{3},\langle\rangle,\right)$ be a minimal immersion of a surface $\Sigma$ in a 3 -dimensional Riemannian manifold $M$. The Jacobi operator of the second variation of the area is the strongly elliptic operator, acting on sections of the normal bundle, $L: \Gamma\left(T^{\perp} \Sigma\right) \rightarrow \Gamma\left(T^{\perp} \Sigma\right)$, given by

$$
L=\Delta^{\perp}+|\sigma|^{2}+\operatorname{Ric}(n),
$$

where $\Delta^{\perp}$ is the normal Laplacian, $\sigma$ is the second fundamental form of $\Phi$, and $\operatorname{Ric}(n)$ is the Ricci curvature of any unit normal vector $n$.

If $\Omega$ is a compact domain of $\Sigma$, the operator $L$, with zero boundary conditions, has discrete spectrum $\lambda_{1}(\Omega)<\lambda_{2}(\Omega)<\cdots \rightarrow \infty$, and the dimension of each eigenspace is finite. The index of $L$ in $\Omega, \operatorname{Index}(L, \Omega)$, is the sum of the dimensions of the eigenspaces corresponding to negative eigenvalues.

The index of the minimal immersion $\Phi: \Sigma \rightarrow M$ is the index of the operator $L$ on $\Sigma$, which is defined by

$$
\operatorname{Index}(\Phi)=\operatorname{Index}(L):=\sup \{\operatorname{Index}(L, \Omega) \mid \Omega \text { compact domain of } \Sigma\} .
$$


The minimal immersion $\Phi$ is called stable if $\operatorname{Index}(\Phi)=0$. This means that the quadratic form $Q: \Gamma_{0}\left(T^{\perp} \Sigma\right) \rightarrow \mathbb{R}$ associated to $L$ is nonnegative,

$$
Q(\eta)=-\int_{\Sigma}\langle L \eta, \eta\rangle d v=\int_{\Sigma}\left\{\left|\nabla^{\perp} \eta\right|^{2}-\left(|\sigma|^{2}+\operatorname{Ric}(n)\right)|\eta|^{2}\right\} d A \geq 0,
$$

for any compactly supported normal section $\eta \in \Gamma_{0}\left(T^{\perp} \Sigma\right)$.

The immersion $\Phi$ is called two-sided if $T^{\perp} \Sigma$ is trivial, i.e., there exists a global unit normal vector field $N$. Otherwise, i.e., when $T^{\perp} \Sigma$ is non trivial, the immersion is called one-sided. When the ambient manifold $M$ is orientable, $\Phi$ is two-sided if and only if $\Sigma$ is orientable. This is not true when $M$ is non orientable.

If $\Phi$ is two-sided, sections of the normal bundle can be identified with functions on the surface in the following way:

$$
\begin{aligned}
\Gamma\left(T^{\perp} \Sigma\right) & \equiv C^{\infty}(\Sigma), \\
\eta & \equiv f, \quad \text { if } \eta=f N,
\end{aligned}
$$

where $N$ is a global unit normal section to $\Phi$. In this case, it is clear that $\Delta^{\perp} \eta=$ $(\Delta f) N$, and hence the Jacobi operator becomes a Schrödinger operator acting on functions $L: C^{\infty}(\Sigma) \rightarrow C^{\infty}(\Sigma)$, given by

$$
L=\Delta+|\sigma|^{2}+\operatorname{Ric}(N)=\Delta-K+\left(|\sigma|^{2}+\rho\right) / 2,
$$

where $K$ is the Gauss curvature of $\Sigma, \rho$ scalar curvature of $M$ and we have used the Gauss equation of $\Phi$ to obtain the second expression of $L$.

When $M$ is the 3 -dimensional unit sphere $\mathbb{S}^{3}$ or the 3 -dimensional real projective space $\mathbb{R P}^{3}$, the Jacobi operator is given by

$$
L=\Delta^{\perp}+|\sigma|^{2}+2,
$$

whereas if $M$ is the Riemannian product $\mathbb{S}^{2} \times \mathbb{R}, \mathbb{R} \mathbb{P}^{2} \times \mathbb{R}$, or one of their quotients $\mathbb{S}^{2} \times \mathbb{S}^{1}(r)$, or $\mathbb{R} \mathbb{P}^{2} \times \mathbb{S}^{1}(r)$, the Jacobi operator is

$$
L=\Delta^{\perp}+|\sigma|^{2}+\left|\xi^{\top}\right|^{2},
$$

where $\mathbb{S}^{1}(r)$ is the circle of radius $r, \xi$ is a unit parallel vertical vector field on the ambient manifold and $T$ stands for the tangential component.

Now, we will recount some background about minimal surfaces, which will be used later. Let $\Phi=(\phi, \psi): \Sigma \rightarrow \mathbb{S}^{2} \times \mathbb{R}$ (respectively $\mathbb{S}^{2} \times \mathbb{S}^{1}(r), \mathbb{R} \mathbb{P}^{2} \times \mathbb{R}$, or $\mathbb{R P}^{2} \times \mathbb{S}^{1}(r)$ ) be a minimal immersion of a surface $\Sigma$ and denote also by $\langle$,$\rangle the$ induced metric. If $\bar{R}$ denotes the curvature operator of the ambient 3-manifold, it is easy to prove that $\bar{R}\left(e_{1}, e_{2}, e_{2}, e_{1}\right)=1-\left|\xi^{\top}\right|^{2}$, where $\left\{e_{1}, e_{2}\right\}$ is an orthonormal basis on $\Sigma$. So, the Gauss equation of $\Phi$ can be written as

$$
K=1-\left|\xi^{\top}\right|^{2}-\frac{|\sigma|^{2}}{2} .
$$

If $\Sigma$ is orientable and $z=x+i y$ is a conformal parameter with induced metric $e^{2 u}|d z|^{2}$, and $\partial_{z}=\left(\partial_{x}-i \partial_{y}\right) / 2$ and $\partial_{\bar{z}}=\left(\partial_{x}+i \partial_{y}\right) / 2$ are the corresponding complex operators, then it is well known that

$$
\Theta(z)=\left\langle\Phi_{z}, \xi\right\rangle d z
$$


is a globally defined holomorphic 1-differential on $\Sigma$. As $|\Theta|^{2}=e^{2 u}\left|\xi^{\top}\right|^{2} / 2$, we have that either $\xi^{\top}=0$ (i.e., $\Theta \equiv 0$ ) or $\left\{p \in \Sigma \mid \xi^{\top}(p)=0\right\}$ is isolated. In the first case, $\xi$ is normal to $\Phi$, and then $d \psi(v)=0$ for any tangent vector $v$, i.e., $\psi$ is constant. Moreover $\phi: \Sigma \rightarrow \mathbb{S}^{2}$ (respectively $\phi: \Sigma \rightarrow \mathbb{R P}^{2}$ ) is a local isometry. In this case we will say that $\Sigma$ is a slice. The Gauss equation says that the slices are totally geodesic surfaces.

Lemma 3.1. Let $\Phi: \Sigma \rightarrow\left(M^{3},\langle\rangle,\right)$ be a minimal immersion of an orientable surface $\Sigma$ and $X$ any harmonic vector field on $\Sigma$.

1) If $M=\mathbb{S}^{3}$, then $L X=2 X+2\langle\sigma, \nabla X\rangle N$,

2) If $M=\mathbb{S}^{2} \times \mathbb{R}$, then

$$
\langle L X, X\rangle=\left(2-\left|\xi^{\top}\right|^{2}\right)\langle X, \xi\rangle^{2},
$$

3) If $M=\mathbb{S}^{2} \times \mathbb{S}^{1}(r)$, then

$$
\langle L X, X\rangle=\left(2-\left(1+\frac{1}{r^{2}}\right)\left|\xi^{\top}\right|^{2}\right)\langle X, \xi\rangle^{2},
$$

where $L$ is the Jacobi operator of the two-sided minimal immersion $\Phi$ and $X$ is considered as an $\mathbb{R}^{4}$-valued function in 1) and 2) and as an $\mathbb{R}^{5}$-valued function in 3 ).

Proof. We consider $\mathbb{S}^{3}, \mathbb{S}^{2} \times \mathbb{R} \subset \mathbb{R}^{4}$ and $\mathbb{S}^{2} \times \mathbb{S}^{1}(r) \subset \mathbb{R}^{5}$. If $\nabla^{0}$ is the connection on $\mathbb{R}^{4}$ or $\mathbb{R}^{5}$ and $\bar{\sigma}$ the second fundamental form of $M$ in $\mathbb{R}^{4}$ or $\mathbb{R}^{5}$, then

$$
\nabla_{v}^{0} X=\nabla_{v} X+\sigma(v, X)+\bar{\sigma}(v, X)
$$

and so, using (2.1),

$$
\begin{aligned}
\Delta^{0} X= & \left(K-|\sigma|^{2} / 2\right) X+2\langle\sigma, \nabla X\rangle N \\
& +\sum_{i=1}^{2}\left\{-\bar{A}_{\bar{\sigma}\left(e_{i}, X\right)} e_{i}+2 \bar{\sigma}\left(e_{i}, \nabla_{e_{i}} X+\sigma\left(e_{i}, X\right)\right)\right\},
\end{aligned}
$$

where $\left\{e_{1}, e_{2}\right\}$ is an orthonormal frame on $\Sigma$ and $\bar{A}$ is the Weingarten endomorphism of $M$ in $\mathbb{R}^{4}$ or $\mathbb{R}^{5}$. Hence,

$$
L X=(\rho / 2) X+2\langle\sigma, \nabla X\rangle N+\sum_{i=1}^{2}\left\{-\bar{A}_{\bar{\sigma}\left(e_{i}, X\right)} e_{i}+2 \bar{\sigma}\left(e_{i}, \nabla_{e_{i}} X+\sigma\left(e_{i}, X\right)\right)\right\} .
$$

Now, using the expressions of the second fundamental forms of these three manifolds in $\mathbb{R}^{4}$ and $\mathbb{R}^{5}$, it is easy to prove the lemma.

Lemma 3.2. Let $\Phi: \Sigma \rightarrow \mathbb{S}^{2} \times \mathbb{R}$ (respectively $\mathbb{S}^{2} \times \mathbb{S}^{1}(r)$ ) be a minimal immersion of an orientable surface $\Sigma$. If $\Phi$ is not a slice, then:

1) The tangential component $\xi^{\top}$ of the vertical vector field $\xi$ is a harmonic vector field on $\Sigma$ with only a discrete number of zeroes. Moreover, the harmonic vector field $J \xi^{\top}$ satisfies $\left\langle J \xi^{\top}, \xi\right\rangle=0$ and any vector field $X$ on $\Sigma$ can be written, almost everywhere, as $X=f \xi^{\top}+g J \xi^{\top}$, for certain functions $f$ and $g$. 
2) If $(\Sigma, \tau) \rightarrow \Sigma_{0}$ is the two-fold oriented covering of a non orientable surface $\Sigma_{0}$ and $\Phi$ is the lift of a minimal immersion $\Phi_{0}: \Sigma_{0} \rightarrow \mathbb{S}^{2} \times \mathbb{R}$ (respectively $\left.\mathbb{S}^{2} \times \mathbb{S}^{1}(r)\right)$, then $\tau_{*} \xi^{\top}=\xi^{\top}$ and $\tau_{*} J \xi^{\top}=-J \xi^{\top}$.

Proof. Using that $\xi$ is a parallel vector field, it is clear that for any $v$ and $w$ tangent to $\Sigma$ we have that

$$
\left\langle\nabla_{v} \xi^{\top}, w\right\rangle=\langle\sigma(v, w), \xi\rangle
$$

This means that $\xi^{\top}$ is a harmonic vector field on $\Sigma$. Also, the harmonic vector field $J \xi^{\top}$ is perpendicular to $\xi$, and so $\left\{\left(\xi^{\top}\right)_{p},\left(J \xi^{\top}\right)_{p}\right\}$ are linearly independent on $\left\{p \in \Sigma \mid \xi^{\top}(p) \neq 0\right\}$. This proves 1$)$.

If $\Pi: \Sigma \rightarrow \Sigma_{0}$ is the projection, then $\Phi=\Phi_{0} \circ \Pi$ and so $\Phi \circ \tau=\Phi$. This implies that $\tau_{*} \xi^{\top}=\xi^{\top}$. Also, as $\tau_{*} \circ J=-J \circ \tau_{*}$, we have that $\tau_{*} J \xi^{\top}=-J \xi^{\top}$.

\section{Statement and proof of the main results}

In this section we will use some results which appear explicitly in [4], [5], and [6], or follow from them. For completeness we recall them here. The next result can be proved using arguments like in Proposition 2 of [4], and so we omit the proof.

Proposition 4.1 ([4]). Let $\Sigma$ be an orientable complete Riemannian surface and let $\tau$ be an isometry of $\Sigma$ without fixed points and with $\tau^{2}=\mathrm{Id}$. Let $L=\Delta+q$ be a Schrödinger operator on $\Sigma$ with $q \circ \tau=q$ and consider the operator

$$
L^{-}=L_{\mid C_{-}^{\infty}(\Sigma)}: C_{-}^{\infty}(\Sigma) \rightarrow C_{-}^{\infty}(\Sigma)
$$

where $C_{-}^{\infty}(\Sigma)=\left\{f \in C^{\infty}(\Sigma) \mid f \circ \tau=-f\right\}$. Then $L^{-}$has finite index $k$ if and only if there exists a $k$-dimensional subspace $W$ of $L_{-}^{2}(\Sigma)$ having an orthonormal basis $\left\{v_{1}, \ldots, v_{k}\right\}$ with $L v_{i}+\lambda_{i} v_{i}=0, \lambda_{i}<0$ and $Q(f) \geq 0$ for any function $f \in C_{0}^{\infty}(\Sigma) \cap W^{\perp}$ with $f \circ \tau=-f$.

Theorem 4.2 ([4], [5], [6]). Let $\Sigma$ be a complete Riemannian surface, let $L=$ $\Delta-K+q$ be a Schrödinger operator on $\Sigma$, where $K$ is the Gauss curvature of $\Sigma$, and suppose $q \geq 0$.

1) If $\Sigma$ is orientable and $\operatorname{Index}(L)=0$, then either $\Sigma$ is conformally equivalent to the sphere $\mathbb{S}^{2}$ or the complex plane $\mathbb{C}$, or $q=0$ and $\Sigma$ is either a flat torus or a flat cylinder.

2) If $q \geq c>0$ for some constant $c$, and there exists a compact set $C \subset \Sigma$ such that $\operatorname{Index}(L)=0$ on $\Sigma-C$, then $\Sigma$ is compact.

From here, we obtain:

Corollary 4.3 ([4], [6]). If $\Phi: \Sigma \rightarrow M^{3}$ is a two-sided minimal immersion of a complete and non compact surface in a Riemannian manifold $M$ with scalar curvature $\rho \geq c>0$, then $\operatorname{Index}(\Phi)=\infty$. 
Proof. As $\Phi$ is two-sided, the Jacobi operator is the Schrödinger operator $L=$ $\Delta-K+\left(\rho+|\sigma|^{2}\right) / 2$. If $\operatorname{Index}(\Phi)<\infty$, from Proposition 1 in [4], there exists a compact set $C \subset \Sigma$ such that $\Sigma-C$ is stable. Now the result follows from 2) of Theorem 4.2.

Using similar ideas, we can extend Corollary 4.3 to a certain family of one-sided complete minimal surfaces.

Corollary 4.4. Let $\Phi: \Sigma \rightarrow M^{3}$ be a one-sided minimal immersion of a complete and non compact surface $\Sigma$ in an orientable Riemannian manifold $M$ with scalar curvature $\rho \geq c>0$. If the genus of the two-fold oriented covering of $\Sigma$ is finite, then $\operatorname{Index}(\Phi)=\infty$.

Remark 4.5. As $\Phi$ is one-sided and $M$ is orientable, $\Sigma$ is not orientable. Also, the orientability of the ambient manifold is necessary in the assumptions, because $\mathbb{R P}^{1} \times \mathbb{R}$ is an orientable complete surface of genus zero, which is embedded in $\mathbb{R P}^{2} \times \mathbb{R}$ as a stable minimal one-sided surface (see Theorem 4.10).

Proof of Corollary 4.4. Let $(\tilde{\Sigma}, \tau)$ be the two-fold oriented covering of $\Sigma$ with $\tau$ the change of sheet on $\tilde{\Sigma}$. As the genus of $\tilde{\Sigma}$ is finite, Lemma 9 in [12] implies that there exists a compact subset $C \subset \Sigma$, such that $\Sigma-C$ is orientable. Hence, as $M$ is orientable, $\Phi: \Sigma-C \rightarrow M$ is a two-sided minimal immersion and so the Jacobi operator on $\Sigma-C$ is $L=\Delta-K+\left(\rho+|\sigma|^{2}\right) / 2$.

If $\operatorname{Index}(\Sigma)$ is finite, then $\operatorname{Index}(\Sigma-C)$ is finite too. So, from Proposition 1 in [4], there exists a compact subset $K \subset \Sigma-C$, such that $\Sigma-(C \cup K)$ is stable. Hence $\Sigma$ is a complete surface and the Schrödinger operator $L=\Delta-K+\left(\rho+|\sigma|^{2}\right) / 2$ on $\Sigma$ satisfies that $\operatorname{Index}(L)=0$ on $\Sigma-(C \cup K)$. By 2) of Theorem 4.2, $\Sigma$ must be compact. This proves the corollary.

Hence, for orientable ambient 3-manifolds with scalar curvature $\rho \geq c>0$, the remaining case to study is when the two-fold oriented covering of the onesided complete and non compact minimal surface has infinite genus. We have not obtained a general result, but following [12] and using harmonic vector fields as test functions, we have found the answer for some particular 3-manifolds.

Theorem 4.6. Let $\Phi: \Sigma \rightarrow M$ be a minimal immersion of a complete surface $\Sigma$ in a 3-dimensional Riemannian manifold $M$.

1) If $M=\mathbb{S}^{3}$ and $\Sigma$ is not compact, then $\operatorname{Index}(\Phi)=\infty$.

2) If $M=\mathbb{S}^{2} \times \mathbb{R}$, then either $\Sigma=\mathbb{S}^{2}, \Phi(\Sigma)=\mathbb{S}^{2} \times\{t\}, t \in \mathbb{R}$ and $\Phi$ is stable, or $\operatorname{Index}(\Phi)=\infty$.

3) If $M=\mathbb{S}^{2} \times \mathbb{S}^{1}(r), r \geq 1$, and $\Sigma$ is not compact, then $\operatorname{Index}(\Phi)=\infty$.

Proof. As the only compact minimal surfaces of $\mathbb{S}^{2} \times \mathbb{R}$ are the slices $\mathbb{S}^{2} \times\{t\}, t \in \mathbb{R}$, which are stable, Corollaries 4.3 and 4.4 imply that we can assume that $\Phi$ is onesided, i.e., $\Sigma$ is not orientable and that the two-fold oriented covering $\tilde{\Sigma}$ of $\Sigma$ has infinite genus. 
Let $\tau$ be the change of sheet in $\tilde{\Sigma}$ and $\Pi: \tilde{\Sigma} \rightarrow \Sigma$ the projection. Then, $\tilde{\Phi}=\Phi \circ \Pi$ is also a minimal immersion which is two-sided. Let $\tilde{N}$ be a global unit vector field normal to $\tilde{\Phi}$. As $\Phi$ is one-sided, $\tilde{N} \circ \tau=-\tilde{N}$. Also, we can identify sections of the normal bundle of $\Phi, \Gamma\left(T^{\perp} \Sigma\right)$, with functions on $\tilde{\Sigma}$ which are odd with respect to $\tau$ :

$$
\begin{aligned}
\Gamma\left(T^{\perp} \Sigma\right) & \equiv C_{-}^{\infty}(\tilde{\Sigma})=\left\{f \in C^{\infty}(\tilde{\Sigma}) \mid f \circ \tau=-f\right\}, \\
\eta & \equiv f, \quad \text { if } \tilde{\eta}=f \tilde{N},
\end{aligned}
$$

where $\tilde{\eta}$ is the lift of $\eta$ to $\tilde{\Phi}$. Also, if $\tilde{L}=\tilde{\Delta}-\tilde{K}+\rho / 2+|\tilde{\sigma}|^{2} / 2$ is the Jacobi operator of $\tilde{\Phi}$, it is clear that

$$
2 Q(\eta)=\tilde{Q}(\tilde{\eta})=\tilde{Q}(f)=-\int_{\tilde{\Sigma}} f \tilde{L} f d \tilde{A}
$$

for any compactly supported $\eta \in \Gamma\left(T^{\perp} \Sigma\right)$.

To prove the result, we suppose that $\operatorname{Index}(\Phi)=k$ and we will find a contradiction.

From Proposition 4.1, there exist functions $\left\{v_{1}, \ldots, v_{k}\right\}$ in $L^{2}(\tilde{\Sigma})$ with $v_{i} \circ \tau=$ $-v_{i}$ and $\tilde{L} v_{i}+\lambda_{i} v_{i}=0, \lambda_{i}<0$, and such that if $f \in C_{0}^{\infty}(\tilde{\Sigma})$ satisfies $f \circ \tau=-f$ and is $L^{2}$-orthogonal to $v_{i}, 1 \leq i \leq k$, then

$$
\tilde{Q}(f) \geq 0 \text {. }
$$

As the genus of $\tilde{\Sigma}$ is infinite, the space $H(\tilde{\Sigma})$ of $L^{2}$-harmonic vector fields on $\tilde{\Sigma}$ has also infinite dimension. Let $V$ be an $l$-dimensional $\left(l>4 k\right.$ if $M=\mathbb{S}^{3}$ or $\mathbb{S}^{2} \times \mathbb{R}$ and $l>5 k$ if $\left.M=\mathbb{S}^{2} \times \mathbb{S}^{1}(r)\right)$ subspace of $H^{-}(\tilde{\Sigma})$. When $M=\mathbb{S}^{2} \times \mathbb{R}$ or $\mathbb{S}^{2} \times \mathbb{S}^{1}(r)$, we will chose $V$ with a different restriction. In fact, in these cases, if the harmonic vector field $J \xi^{\top}$ (see Lemma 3.2) satisfies $\int_{\tilde{\Sigma}}\left|J \xi^{\top}\right|^{2}<\infty$, i.e., $J \xi^{\top} \in H^{-}(\tilde{\Sigma})$, we will take $V$ to be $L_{2}$-orthogonal to the line spanned by $J \xi^{\top}$. If $\int_{\tilde{\Sigma}}\left|J \xi^{\top}\right|^{2}=\infty$, we will impose no further restrictions on $V$.

Let $\left\{U_{n} \mid n \in \mathbb{N}\right\}$ be an exhaustion of the complete surface $\tilde{\Sigma}$ and $\left\{\varphi_{n} \mid n \in \mathbb{N}\right\}$ cut-off functions on $\tilde{\Sigma}$ with $\operatorname{supp}\left(\varphi_{n}\right) \subset U_{n},\left|\nabla \varphi_{n}\right|^{2} \leq 1$, and $\varphi_{n} \circ \tau=\varphi_{n}$.

For each $n \in \mathbb{N}$, let $F_{n}: V \rightarrow \mathbb{R}^{4 k}$ (respectively $\mathbb{R}^{5 k}$ ) be the linear map given by

$$
F_{n}(X)=\left(\int_{\tilde{\Sigma}} \varphi_{n} v_{1} X, \ldots, \int_{\tilde{\Sigma}} \varphi_{n} v_{k} X\right) .
$$

As $l>4 k$ (respectively $l>5 k$ ), we can choose $X_{n} \in \operatorname{ker} F_{n}$ with $\int_{\tilde{\Sigma}}\left|X_{n}\right|^{2}=1$. So, for each $n$, the function $\varphi_{n} X_{n}$ has compact support, is $L^{2}$-orthogonal to $v_{i}, 1 \leq$ $i \leq k$ and, as $X_{n} \in H^{-}(\tilde{\Sigma})$, we have that $\varphi_{n} X_{n} \circ \tau=-\varphi_{n} X_{n}$. Hence

$$
\tilde{Q}\left(\varphi_{n} X_{n}\right) \geq 0, \quad \forall n \in \mathbb{N} .
$$

Now,

$$
\tilde{Q}\left(\varphi_{n} X_{n}\right)=-\int_{\tilde{\Sigma}} \varphi_{n} \tilde{\Delta} \varphi_{n}\left|X_{n}\right|^{2}-\int_{\tilde{\Sigma}} \frac{1}{2}\left\langle\tilde{\nabla} \varphi_{n}^{2}, \tilde{\nabla}\left|X_{n}\right|^{2}\right\rangle-\int_{\tilde{\Sigma}} \varphi_{n}^{2}\left\langle\tilde{L} X_{n}, X_{n}\right\rangle .
$$


As $\operatorname{div}\left(\left|X_{n}\right|^{2} \tilde{\nabla} \varphi_{n}^{2}\right)=\left\langle\tilde{\nabla} \varphi_{n}^{2}, \tilde{\nabla}\left|X_{n}\right|^{2}\right\rangle+2\left(\varphi_{n} \tilde{\Delta} \varphi_{n}+\left|\tilde{\nabla} \varphi_{n}\right|^{2}\right)\left|X_{n}\right|^{2}$, we finally obtain

$$
\int_{\tilde{\Sigma}} \varphi_{n}^{2}\left\langle\tilde{L} X_{n}, X_{n}\right\rangle \leq \int_{\tilde{\Sigma}}\left|\tilde{\nabla} \varphi_{n}\right|^{2}\left|X_{n}\right|^{2}, \quad \forall n \in \mathbb{N} .
$$

Using Lemma 3.1, we get

$$
\int_{\tilde{\Sigma}}\left|\tilde{\nabla} \varphi_{n}\right|^{2}\left|X_{n}\right|^{2} \geq\left\{\begin{array}{l}
2 \int_{\tilde{\Sigma}} \varphi_{n}^{2}\left|X_{n}\right|^{2}, \text { when } M=\mathbb{S}^{3}, \\
\int_{\tilde{\Sigma}} \varphi_{n}^{2}\left(2-\left|\xi^{\top}\right|^{2}\right)\left\langle X_{n}, \xi\right\rangle^{2}, \text { when } M=\mathbb{S}^{2} \times \mathbb{R}, \\
\int_{\tilde{\Sigma}} \varphi_{n}^{2}\left(2-\left(1+\frac{1}{r^{2}}\right)\left|\xi^{\top}\right|^{2}\right)\left\langle X_{n}, \xi\right\rangle^{2}, \text { when } M=\mathbb{S}^{2} \times \mathbb{S}^{1}(r) .
\end{array}\right.
$$

It is clear that the sequence of harmonic vector fields $X_{n}$ converges in $V$, up to extraction of a subsequence, to a harmonic vector field $X$ with $\int_{\tilde{\Sigma}}|X|^{2}=1$. Using the remark made at the end of Section 2, we have that

$$
\lim \int_{\tilde{\Sigma}}\left|\nabla \varphi_{n}\right|^{2}\left|X_{n}\right|^{2}=\int_{\tilde{\Sigma}} \lim \left|\nabla \varphi_{n}\right|^{2}\left|X_{n}\right|^{2}=0,
$$

because $\left|\nabla \varphi_{n}\right|^{2} \rightarrow 0$. So, when $M=\mathbb{S}^{3}$,

$$
0=\lim \int_{\tilde{\Sigma}} \varphi_{n}^{2}\left|X_{n}\right|^{2}=\int_{\tilde{\Sigma}} \lim \varphi_{n}^{2}\left|X_{n}\right|^{2}=\int_{\tilde{\Sigma}}|X|^{2}=1,
$$

which is a contradiction. When $M=\mathbb{S}^{2} \times \mathbb{R}$, we have

$$
\begin{aligned}
0 & =\lim \int_{\tilde{\Sigma}} \varphi_{n}^{2}\left(2-\left|\xi^{\top}\right|^{2}\right)\left\langle X_{n}, \xi\right\rangle^{2} \\
& =\int_{\tilde{\Sigma}} \lim \varphi_{n}^{2}\left(2-\left|\xi^{\top}\right|^{2}\right)\left\langle X_{n}, \xi\right\rangle^{2}=\int_{\tilde{\Sigma}}\left(2-\left|\xi^{\top}\right|^{2}\right)\langle X, \xi\rangle^{2},
\end{aligned}
$$

which implies that $\langle X, \xi\rangle=0$. When $M=\mathbb{S}^{2} \times \mathbb{S}^{1}(r)$, we have

$$
0=\lim \int_{\tilde{\Sigma}} \varphi_{n}^{2}\left(2-\left(1+\frac{1}{r^{2}}\right)\left|\xi^{\top}\right|^{2}\right)\left\langle X_{n}, \xi\right\rangle^{2}=\int_{\tilde{\Sigma}}\left(2-\left(1+\frac{1}{r^{2}}\right)\left|\xi^{\top}\right|^{2}\right)\langle X, \xi\rangle^{2} .
$$

As $r \gtrless 1$, we have that either $r=1$ and $\left|\xi^{\top}\right|^{2}=1$ or $\langle X, \xi\rangle=0$. In the first case, $\tilde{\Sigma}$ is a covering of the totally geodesic torus $\mathbb{S}^{1} \times \mathbb{S}^{1}$, and so it genus cannot be infinite. So, $\langle X, \xi\rangle=0$.

Hence, in the last two cases, using Lemma 3.2, we obtain that the harmonic vector field $X \in V$ is given by $X=\lambda J \xi^{\top}$ for a certain nonzero real number $\lambda$. Hence, $1=\lambda^{2} \int_{\tilde{\Sigma}}\left|J \xi^{\top}\right|^{2}$ and so $J \xi^{\top} \in H^{-}(\tilde{\Sigma})$. As, in this case, $J \xi^{\top}$ is orthogonal to $V$, we get again a contradiction. This finishes the proof.

Now, we extend the results of Theorem 4.6 to minimal surfaces in the real projective space. Although we use a similar idea, the proof is more complicated and in it we will use different test functions.

Theorem 4.7. Let $\Phi: \Sigma \rightarrow \mathbb{R P}^{3}$ be a minimal immersion of a complete non compact surface. Then $\operatorname{Index}(\Phi)=\infty$. 
Proof. Following reasoning as in the previous theorem, we can assume that $\Phi$ is one-sided, i.e., $\Sigma$ is not orientable, and that the two-fold oriented covering $(\tilde{\Sigma}, \tau)$ of $\Sigma$ has infinite genus. Then, $\tilde{\Phi}=\Phi \circ \Pi$ is a two-sided minimal immersion of $\tilde{\Sigma}$ into $\mathbb{R P}^{3}$. We consider two cases.

First case: $\tilde{\Phi}$ admits a lift $\Psi$ to $\mathbb{S}^{3}$.

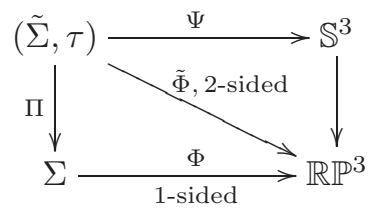

In this case we can identify sections $\Gamma\left(T^{\perp} \Sigma\right)$ of the normal bundle to $\Phi$ with functions on $\tilde{\Sigma}$ which are odd with respect to $\tau$ :

$$
\begin{aligned}
\Gamma\left(T^{\perp} \Sigma\right) & \equiv C_{-}^{\infty}(\tilde{\Sigma}), \\
\eta & \equiv f, \quad \text { if } \quad \tilde{\eta}=f \tilde{N},
\end{aligned}
$$

where $\tilde{\eta}$ is the lift of $\eta$ to $\Psi$. Moreover the quadratic forms associated to $\Phi$ and $\Psi$ satisfy

$$
2 Q(\eta)=\tilde{Q}(\tilde{\eta})=\tilde{Q}(f)
$$

for any compactly supported $f \in C_{-}^{\infty}(\tilde{\Sigma})$. Now, the immersion $\Psi$ is subject to the same conditions that the immersion $\tilde{\Phi}$ in 1 ) of Theorem 4.6. So the result follows making the same proof than in 1) of Theorem 4.6.

Second case: $\tilde{\Phi}$ cannot be lifted to $\mathbb{S}^{3}$. Then, the two-fold covering $\mathbb{S}^{3} \rightarrow \mathbb{R} \mathbb{P}^{3}$ induces a two-fold covering $\hat{\Sigma} \rightarrow \tilde{\Sigma}$ of a connected surface $\hat{\Sigma}$. If $\hat{\tau}$ is the change of sheet, then $\hat{\Phi} \circ \hat{\tau}=-\hat{\Phi}$ and $\hat{\Phi}$ is two-sided.

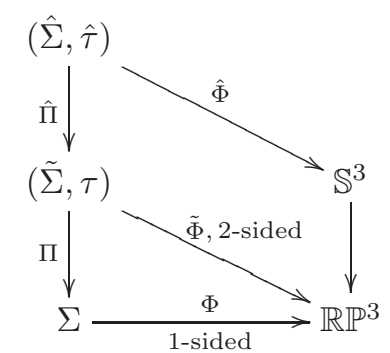

Now, we can lift $\tau$ to $\hat{\Sigma}$ as follows: given $x \in \hat{\Sigma}, \tau(x)=y$ if $\tau(\hat{\Pi}(x))=\hat{\Pi}(y)$ and $\hat{\Phi}(x)=\hat{\Phi}(y)$. As $y$ is uniquely determined, the lift of $\tau$ to $\hat{\Sigma}$, which will be denoted also by $\tau$, is well defined.

Let $N$ be a unit vector field normal to $\hat{\Phi}$. As $\tilde{\Phi}$ is two-sided and $\Phi$ is one-sided, then $N \circ \tau=-N$. Also, as $N$ projects to $\tilde{\Phi}$, then $N \circ \hat{\tau}=-N$.

Now, we can identify normal sections of $\Phi$ with functions on $\hat{\Sigma}$ with the following symmetries:

$$
\begin{aligned}
\Gamma\left(T^{\perp} \Sigma\right) & \equiv C_{ \pm}^{\infty}(\hat{\Sigma})=\left\{f \in C^{\infty}(\hat{\Sigma}) \mid f \circ \hat{\tau}=f, f \circ \tau=-f\right\} \\
\eta & \equiv f, \quad \hat{\eta}=f N,
\end{aligned}
$$


where $\hat{\eta}$ is the lift of $\eta$ to $\hat{\Phi}$. It is clear that $\hat{\eta} \circ \tau=-\hat{\eta} \circ \hat{\tau}=\hat{\eta}$, and so the function $f$ satisfies $f \circ \hat{\tau}=-f \circ \tau=f$.

If $Q$ and $\hat{Q}$ are the quadratic forms associated to the minimal immersions $\Phi$ and $\hat{\Phi}$,

$$
4 Q(\eta)=\hat{Q}(\hat{\eta})=\hat{Q}(f)=-\int_{\hat{\Sigma}} f \hat{L} f
$$

for any compactly supported function $f \in C_{ \pm}^{\infty}(\hat{\Sigma})$, where $\hat{L}: C_{ \pm}^{\infty}(\hat{\Sigma}) \rightarrow C_{ \pm}^{\infty}(\hat{\Sigma})$ is given by

$$
\hat{L}=\hat{\Delta}-\hat{K}+\left(3+|\hat{\sigma}|^{2} / 2\right) .
$$

To prove the result, we suppose that $\operatorname{Index}(\Phi)=k$ and we will find a contradiction.

From Proposition 4.1, there exist functions $\left\{v_{1}, \ldots, v_{k}\right\}$ in $L^{2}(\hat{\Sigma})$ with $v_{i} \circ \tau=$ $-v_{i}, v_{i} \circ \hat{\tau}=v_{i}$ and $\hat{L} v_{i}+\lambda_{i} v_{i}=0, \lambda_{i}<0$, and such that if $f$ is a compactly supported function in $C_{ \pm}^{\infty}(\hat{\Sigma})$ which is $L^{2}$-orthogonal to $v_{i}, 1 \leq i \leq k$, then

$$
\hat{Q}(f) \geq 0
$$

As the genus of $\hat{\Sigma}$ is infinite, the space $H(\hat{\Sigma})$ of $L^{2}$-harmonic vector fields on $\hat{\Sigma}$ has also infinite dimension. Let $V$ be an $l$-dimensional $(l>16 k)$ subspace of $H(\hat{\Sigma})$ such that $\tau_{*} X=-X$ and $\hat{\tau}_{*} X=X$ for any $X \in V$. Let $Y$ be any compactly supported function in $C_{ \pm}^{\infty}\left(\hat{\Sigma}, \mathbb{R}^{4}\right)$ which is $L^{2}$-orthogonal to $v_{j}, j=1,2,3,4$. Let $\left\{U_{n} \mid n \in \mathbb{N}\right\}$ be an exhaustion of the complete surface $\hat{\Sigma}$ and let $\left\{\varphi_{n} \mid n \in \mathbb{N}\right\}$ be cut-off functions on $\hat{\Sigma}$ satisfying $\operatorname{supp}(Y) \subset \overline{\left\{p \in \tilde{\Sigma} \mid \varphi_{n}(p)=1\right\}}, \operatorname{supp}\left(\varphi_{n}\right) \subset U_{n}$, $\left|\nabla \varphi_{n}\right|^{2} \leq 1$, and $\varphi_{n} \circ \tau=\varphi_{n} \circ \hat{\tau}=\varphi_{n}$.

Let $\left\{a_{1}, \ldots, a_{4}\right\}$ be an orthonornal basis of $\mathbb{R}^{4}$. For each $n \in \mathbb{N}$, let $F_{n}: V \rightarrow \mathbb{R}^{16 k}$ be the linear map given by

$$
F_{n}(X)=\left(\int_{\hat{\Sigma}} \varphi_{n} v_{i}\left\langle\hat{\Phi}, a_{j}\right\rangle X\right)_{i j} .
$$

As $l>16 k$, there exists $X_{n} \in \operatorname{ker} F_{n}$ with $\int_{\hat{\Sigma}}\left|X_{n}\right|^{2}=1$. So, for each $n$ and each $j \in\{1,2,3,4\}$, the functions $\varphi_{n}\left\langle\hat{\Phi}, a_{j}\right\rangle X_{n}$ have compact support and they are $L^{2}$-orthogonal to $v_{i}, 1 \leq i \leq k$. Using the properties of $X_{n}$ and $\varphi_{n}$ with respect to $\tau$ and $\hat{\tau}$ and the fact that $\hat{\Phi} \circ \tau=\hat{\Phi}$ and $\hat{\Phi} \circ \hat{\tau}=-\hat{\Phi}$, we obtain that $\varphi_{n}\left\langle\hat{\Phi}, a_{j}\right\rangle X_{n} \circ \tau=-\varphi_{n}\left\langle\hat{\Phi}, a_{j}\right\rangle X_{n} \circ \hat{\tau}=-\varphi_{n}\left\langle\hat{\Phi}, a_{j}\right\rangle X_{n}$.

Now, for each $t \in \mathbb{R}$, let $F_{t}=\varphi_{n}\left\langle\hat{\Phi}, a_{j}\right\rangle X_{n}+t Y$. Then $F_{t} \in C_{ \pm}^{\infty}\left(\hat{\Sigma}, \mathbb{R}^{4}\right)$, has compact support and is $L^{2}$-orthogonal to $v_{i}, 1 \leq i \leq k$. Hence

$$
0 \leq \hat{Q}\left(F_{t}\right), \quad \forall t \in \mathbb{R} .
$$

This means that

$$
\hat{Q}\left(\left\langle\hat{\Phi}, a_{j}\right\rangle X_{n}, Y\right)^{2} \leq \hat{Q}(Y) \hat{Q}\left(\varphi_{n}\left\langle\hat{\Phi}, a_{j}\right\rangle X_{n}\right), \quad \hat{Q}(Y) \geq 0, \quad 1 \leq j \leq 4 .
$$

Making a computation like in the proof of the above theorem, we obtain

$$
\hat{Q}\left(\varphi_{n}\left\langle\hat{\Phi}, a_{j}\right\rangle X_{n}\right)=\int_{\hat{\Sigma}}\left|\hat{\nabla} \varphi_{n}\right|^{2}\left\langle\hat{\Phi}, a_{j}\right\rangle^{2}\left|X_{n}\right|^{2}-\int_{\hat{\Sigma}} \varphi_{n}^{2}\left\langle\hat{L}\left(\left\langle\hat{\Phi}, a_{j}\right\rangle X_{n}\right),\left\langle\hat{\Phi}, a_{j}\right\rangle X_{n}\right\rangle,
$$


for all $n \in \mathbb{N}$ and $1 \leq j \leq 4$. Now, using Lemma 3.1 it is not difficult to see that

$$
\begin{aligned}
\left\langle\hat{L}\left(\left\langle\hat{\Phi}, a_{j}\right\rangle X_{n}\right),\left\langle\hat{\Phi}, a_{j}\right\rangle X_{n}\right\rangle= & \left\langle\hat{\Phi}, a_{j}\right\rangle \hat{\Delta}\left\langle\hat{\Phi}, a_{j}\right\rangle\left|X_{n}\right|^{2}+\left\langle\hat{\Phi}, a_{j}\right\rangle^{2}\left\langle\hat{L} X_{n}, X_{n}\right\rangle \\
& +\frac{1}{2}\left\langle\hat{\nabla}\left\langle\hat{\Phi}, a_{j}\right\rangle^{2}, \hat{\nabla}\left|X_{n}\right|^{2}\right\rangle=\frac{1}{2}\left\langle\hat{\nabla}\left\langle\hat{\Phi}, a_{j}\right\rangle^{2}, \hat{\nabla}\left|X_{n}\right|^{2}\right\rangle
\end{aligned}
$$

However, $\sum_{j=1}^{4}\left\langle\hat{\nabla}\left\langle\hat{\Phi}, a_{j}\right\rangle^{2}, \hat{\nabla}\left|X_{n}\right|^{2}\right\rangle=0$, and so

$$
\sum_{j=1}^{4} \hat{Q}\left(\varphi_{n}\left\langle\hat{\Phi}, a_{j}\right\rangle X_{n}\right)=\int_{\hat{\Sigma}}\left|\nabla \varphi_{n}\right|^{2}\left|X_{n}\right|^{2} .
$$

Using an argument similar to that in the proof of Theorem 4.6 we obtain

$$
\lim \int_{\hat{\Sigma}}\left|\nabla \varphi_{n}\right|^{2}\left|X_{n}\right|^{2}=0
$$

and so from (4.3) we get

$$
0=\sum_{j=1}^{4} \lim \hat{Q}\left(\left\langle\hat{\Phi}, a_{j}\right\rangle X_{n}, Y\right)^{2}=\sum_{j=1}^{4} \hat{Q}\left(\left\langle\hat{\Phi}, a_{j}\right\rangle X, Y\right)^{2},
$$

where $X=\lim X_{n} \in V$, after extracting a subsequence. As $Y$ is arbitrary, we finally get that $\hat{L}(\langle\hat{\Phi}, a\rangle X)=0, \forall a \in \mathbb{R}^{4}$. In particular, using Lemma 3.1 we deduce that $0=\langle\hat{L}(\langle\hat{\Phi}, a\rangle X), \hat{\Phi}\rangle=-2\langle X, a\rangle$, which is a contradiction.

In [15], Torralbo and Urbano classified the compact stable minimal submanifolds of the product of two spheres. As a particular case of that classification, the authors obtain that the slices $\mathbb{S}^{2} \times\{p\}, p \in \mathbb{S}^{1}(r)$ are the only compact stable minimal surfaces in $\mathbb{S}^{2} \times \mathbb{S}^{1}(r)$. In the next result we study the index of a compact minimal surface of $\mathbb{S}^{2} \times \mathbb{S}^{1}(r)$.

Theorem 4.8. Let $\Phi: \Sigma \rightarrow \mathbb{S}^{2} \times \mathbb{S}^{1}(r), r \geq 1$, be a minimal immersion of a compact surface $\Sigma$.

1) If $\Sigma$ is orientable of genus $g$, then $\operatorname{Index}(\Phi) \geq \frac{2 g-1}{5}$. Moreover, Index $(\Phi)=1$ if and only if $r=1$ and $\Phi$ is the totally geodesic embedding $\mathbb{S}^{1} \times \mathbb{S}^{1} \subset \mathbb{S}^{2} \times \mathbb{S}^{1}$.

2) If $\Sigma$ is nonorientable, then Index $(\Phi) \geq \frac{g-1}{5}$, where $g$ is the genus of the two-fold oriented covering of $\Sigma$.

Remark 4.9. Note that when $r>1$, there are no compact orientable minimal surfaces of index one in $\mathbb{S}^{2} \times \mathbb{S}^{1}(r)$. Also, the idea used in the proof does not work for $r<1$. In the case where $r<1$, not only the totally geodesic embedding $\mathbb{S}^{1} \times \mathbb{S}^{1}(r) \subset$ $\mathbb{S}^{2} \times \mathbb{S}^{1}(r)$ has index one, but also any $m$-sheeted covering of $\mathbb{S}^{1} \times \mathbb{S}^{1}(m r) \rightarrow$ $\mathbb{S}^{1} \times \mathbb{S}^{1}(r)$ with $m \leq 1 / r$ has index one.

Proof. First we prove that Index $(\Phi) \geq \frac{2 g-1}{5}$. When $g=0$ the above inequality is irrelevant and when $g=1$ is known, because the only stable compact minimal surfaces of $\mathbb{S}^{2} \times \mathbb{S}^{1}(r)$ have genus zero. So we can assume that $g \geq 2$. Let $m=\operatorname{Index}(\Phi)$. As in this case the immersion is two-sided, the Jacobi operator $L$ acts on functions. Let $\left\{\varphi_{1}, \ldots, \varphi_{m}\right\}$ be eigenfunctions of $L$ corresponding to the negative eigenvalue $\lambda_{1}, \ldots, \lambda_{m}$. Considering $\mathbb{S}^{2} \times \mathbb{S}^{1}(r) \subset \mathbb{R}^{5}$ and an orthonormal 
frame $\left\{a_{1}, \ldots, a_{5}\right\}$ in $\mathbb{R}^{5}$, we define the linear function $F: H(\Sigma) \rightarrow \mathbb{R}^{5 m}$ by

$$
F(X)=\left(\int_{\Sigma} \varphi_{1} X, \ldots, \int_{\Sigma} \varphi_{m} X\right),
$$

where $X$ is considered as an $\mathbb{R}^{5}$-valued function.

Suppose that $X \in \operatorname{ker} F$. Then $X$ is $L_{2}$-orthogonal to each $\varphi_{i}, 1 \leq i \leq m$ and hence $Q(X) \geq 0$. From Lemma 3.1, we have that

$$
0 \leq Q(X)=-\int_{\Sigma}\langle X, \xi\rangle^{2}\left[2-\left(1+\frac{1}{r^{2}}\right)\left|\xi^{\top}\right|^{2}\right] \leq 0,
$$

where the last inequality holds because we suppose $r^{2} \geq 1$. This implies that either $r=1$ and $\left|\xi^{\top}\right|^{2}=1$, or $\langle X, \xi\rangle=0$. In the first case, $\Sigma$ is a finite covering of the totally geodesic torus $\mathbb{S}^{1} \times \mathbb{S}^{1}$, and so it has genus one, which is not the case. Hence $\langle X, \xi\rangle=0$, and from Lemma 3.2, $X=\lambda J \xi^{\top}$, with $\lambda \in \mathbb{R}$, that is, $\operatorname{dim} \operatorname{ker} F \leq 1$. As $2 g=\operatorname{dim} H(\Sigma)=\operatorname{dim} \operatorname{ker} F+\operatorname{dim} \operatorname{Im} F \leq 1+5 m$, we get the result.

If $\Sigma$ is compact and nonorientable, let $\tilde{\Sigma}$ be the two-fold oriented covering of $\Sigma$ and $\tau$ the change of sheet in $\tilde{\Sigma}$. Using the same argument as in previous results, the index of $\Phi$ is the index of the Schrödinger operator $\tilde{L}=\tilde{\Delta}-\tilde{K}+1+|\tilde{\sigma}|^{2} / 2$ acting on $C_{-}^{\infty}(\tilde{\Sigma})=\left\{f \in C^{\infty}(\tilde{\Sigma}) \mid f \circ \tau=-f\right\}$. From Proposition 4.1, let $\left\{\varphi_{1}, \ldots, \varphi_{m}\right\}$ be eigenfunctions of $\tilde{L}$, with $\varphi_{i} \circ \tau=-\varphi_{i}$, corresponding to the negative eigenvalues $\lambda_{1}, \ldots, \lambda_{m}$, where $m$ is the index of $\Phi$. In this case, we define the linear map $F: H^{-}(\tilde{\Sigma}) \rightarrow \mathbb{R}^{5 m}$ by

$$
F(X)=\left(\int_{\tilde{\Sigma}} \varphi_{1} X, \ldots, \int_{\tilde{\Sigma}} \varphi_{m} X\right)
$$

where $X$ is considered as an $\mathbb{R}^{5}$-valued function. Following the same idea as above and taking into account that $\operatorname{dim} H^{-}(\tilde{\Sigma})=g$, we prove 2$)$.

Finally, it is easy to check that the totally geodesic embedding $\mathbb{S}^{1} \times \mathbb{S}^{1}(r) \subset$ $\mathbb{S}^{2} \times \mathbb{S}^{1}(r), r \geq 1$, has index one if and only if $r=1$.

Conversely, let $\Phi: \Sigma \rightarrow \mathbb{S}^{2} \times \mathbb{S}^{1}(r), r \geq 1$, be a minimal immersion of an orientable and compact surface $\Sigma$ with index one. If $\Phi=(\phi, \psi)$, for each $a \in \mathbb{R}^{2}$ the function $\langle\psi, a\rangle: \Sigma \rightarrow \mathbb{R}$ satisfies

$$
v(\langle\psi, a\rangle)=\left\langle\psi_{*}(v), a\right\rangle=\langle v, \xi\rangle\langle\xi, a\rangle .
$$

Hence

$$
\nabla\langle\psi, a\rangle=\langle\xi, a\rangle \xi^{\top}
$$

Hence the Laplacian of $\langle\psi, a\rangle$ is given by

$$
\Delta\langle\psi, a\rangle=\left\langle\bar{\sigma}\left(\xi^{\top}, \xi\right), a\right\rangle+\langle\xi, a\rangle \operatorname{div} \xi^{\top}=-\frac{1}{r^{2}}\left\langle\xi^{\top}, \xi\right\rangle\langle\psi, a\rangle=-\frac{\left|\xi^{\top}\right|^{2}}{r^{2}}\langle\psi, a\rangle .
$$

Therefore, the Jacobi operator of the surface $\Sigma$ acting on $\langle\psi, a\rangle$ is given by

$$
L\langle\psi, a\rangle=\left(\left(1-1 / r^{2}\right)\left|\xi^{\top}\right|^{2}+|\sigma|^{2}\right)\langle\psi, a\rangle,
$$

and

$$
Q(\langle\psi, a\rangle)=-\int_{\Sigma}\left(\left(1-1 / r^{2}\right)\left|\xi^{\top}\right|^{2}+|\sigma|^{2}\right)\langle\psi, a\rangle^{2} d A \leq 0 .
$$


If $V=\left\{\langle\psi, a\rangle, a \in \mathbb{R}^{2}\right\}$ and $\operatorname{dim} V \leq 1$, then there exists a non zero vector $a$ in $\mathbb{R}^{2}$ such that $\langle\psi, a\rangle=0$ and so $\psi$ is constant. Hence $\Phi(\Sigma)$ is a slice of $\mathbb{S}^{2} \times \mathbb{S}^{1}(r)$, which is stable. So $\operatorname{dim} V=2$. Now, as the index of $\Sigma$ is one and $\xi^{\top}$ has only isolated zeroes, one gets that $\sigma=0$ and $r=1$. Then, $\Phi(\Sigma)$ is a finite covering of the totally geodesic surface $\mathbb{S}^{1} \times \mathbb{S}^{1}$. As the index is one, the surface must be $\mathbb{S}^{1} \times \mathbb{S}^{1}$ and the proof of 1 ) is complete.

To finish, we will take $\mathbb{R} \mathbb{P}^{2} \times \mathbb{R}$ as the ambient manifold. This 3-manifold is non orientable (its two-fold oriented covering is $\mathbb{S}^{2} \times \mathbb{R}$ ) and so the two-sidedness of its immersed surfaces does not follow from their orientability. In any case, from Corollary 4.3, a two-sided stable complete minimal surface of $\mathbb{R P}^{2} \times \mathbb{R}$ must be compact and then the surface is a slice $\mathbb{R P}^{2} \times\{t\}$, which is stable. When the minimal surface $\Sigma$ is one-sided, $\Sigma$ can be orientable or non orientable. In the first case we classify the stable ones. Hence, the classification of stable one-sided complete minimal surfaces of $\mathbb{R P}^{2} \times \mathbb{R}$ is still open for the non orientable ones.

Theorem 4.10. Let $\Phi: \Sigma \rightarrow \mathbb{R P}^{2} \times \mathbb{R}$ be a minimal immersion of an orientable complete surface. Then $\Phi$ is stable if and only if

1) $\Sigma=\mathbb{S}^{2}, \Phi(\Sigma)=\mathbb{R} \mathbb{P}^{2} \times\{t\}, t \in \mathbb{R}$, or

2) $\Phi$ is the totally geodesic embedding of $\mathbb{R P}^{1} \times \mathbb{R}$ into $\mathbb{R} \mathbb{P}^{2} \times \mathbb{R}$.

Remark 4.11. Note that the stable totally geodesic embedding $\mathbb{R P}^{1} \times \mathbb{R} \subset \mathbb{R P}^{2} \times \mathbb{R}$ is the quotient, under the projection $\mathbb{S}^{2} \times \mathbb{R} \rightarrow \mathbb{R P}^{2} \times \mathbb{R}$, of the totally geodesic embedding $\mathbb{S}^{1} \times \mathbb{R} \subset \mathbb{S}^{2} \times \mathbb{R}$ whose index is infinite.

Proof. It is clear that the totally geodesic immersion of $\mathbb{S}^{2}$ into $\mathbb{R P}^{2} \times \mathbb{R}$ given in 1 ) is stable. Also, as the totally geodesic embedding $\mathbb{R P}^{1} \subset \mathbb{R P}^{2}$ is a stable geodesic, it is not difficult to check that the embedding given in 2) is also stable.

Conversely, we suppose that $\Phi$ is stable. If $\Phi$ is two-sided, by Corollary 4.3, $\Sigma$ is compact. As $\Sigma$ is orientable, we obtain that $\Sigma=\mathbb{S}^{2}$ and $\Phi(\Sigma)=\mathbb{R P}^{2} \times\{t\}$.

If $\Phi$ is one-sided then $\Sigma$ is non compact, because the only two compact examples are two-sided.

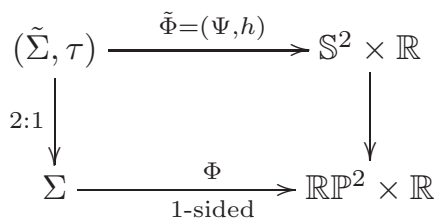

Let $\mathbb{S}^{2} \times \mathbb{R} \rightarrow \mathbb{R P}^{2} \times \mathbb{R}$ be the projection. As $\Sigma$ is orientable, $\Phi$ does not lift to $\mathbb{S}^{2} \times \mathbb{R}$, and so the above covering induces a two-fold covering $\tilde{\Sigma} \rightarrow \Sigma$ of a connected surface $\tilde{\Sigma}$. If $\tau$ is the change of sheet and $\tilde{\Phi}=(\Psi, h): \tilde{\Sigma} \rightarrow \mathbb{S}^{2} \times \mathbb{R}$ the corresponding minimal immersion, then $\Psi \circ \tau=-\Psi$ and $\tilde{\Phi}$ is two-sided.

Now we can identify sections of the normal bundle of $\Phi$ with functions on $\tilde{\Sigma}$ which are odd with respect to $\tau$ in the following way:

$$
\begin{aligned}
\Gamma\left(T^{\perp} \Sigma\right) & \equiv C_{-}^{\infty}(\tilde{\Sigma})=\left\{f \in C^{\infty}(\tilde{\Sigma}) \mid f \circ \tau=-f\right\} \\
\eta & \equiv f, \quad \text { if } \tilde{\eta}=f N,
\end{aligned}
$$


where $\tilde{\eta}$ is the lift of $\eta$ to $\tilde{\Phi}$ and $N$ is a unit vector field normal to $\tilde{\Phi}$. As $\Phi$ is one-sided, then $N \circ \tau=-N$ and so, $f \circ \tau=-f$, because $\tilde{\eta} \circ \tau=\tilde{\eta}$.

Moreover, it is easy to check that if $\eta \in \Gamma_{0}\left(T^{\perp} \Sigma\right)$ and $f \in C_{-}^{\infty}(\tilde{\Sigma})$ is the corresponding function with $\tilde{\eta}=f N$, then

$$
2 Q(\eta)=\tilde{Q}(\tilde{\eta})=\tilde{Q}(f)=-\int_{\tilde{\Sigma}} f \tilde{L} f
$$

where $\tilde{L}: C_{-}^{\infty}(\tilde{\Sigma}) \rightarrow C_{-}^{\infty}(\tilde{\Sigma})$ is given by

$$
\tilde{L}=\tilde{\Delta}-\tilde{K}+\left(1+|\tilde{\sigma}|^{2} / 2\right) .
$$

Given $\varphi \in C_{0}^{\infty}(\Sigma)$, its lift $\tilde{\varphi} \in C_{0}^{\infty}(\tilde{\Sigma})$ satisfies $\tilde{\varphi} \circ \tau=\tilde{\varphi}$. Then, the $\mathbb{R}^{3}$-valued function $\tilde{\varphi} \Psi\left(\Psi: \tilde{\Sigma} \rightarrow \mathbb{S}^{2}, \Psi \circ \tau=-\Psi\right)$ has compact support and $\tilde{\varphi} \Psi \circ \tau=-\tilde{\varphi} \Psi$. As $\Phi$ is stable,

$$
0 \leq \tilde{Q}(\tilde{\varphi} \Psi)
$$

Now we compute $\tilde{Q}(\tilde{\varphi} \Psi)$. First, $\tilde{L}(\tilde{\varphi} \Psi)=(\tilde{\Delta} \tilde{\varphi}) \Psi+\tilde{\varphi} \tilde{L} \Psi+2 \nabla_{\tilde{\nabla} \tilde{\varphi}}^{E} \Psi$, where $\nabla^{E}$ is the connection on $\mathbb{R}^{4}$.

First, $\tilde{\Delta} \Psi=-\left(\sum_{i=1}^{2}\left|\Psi_{*} e_{i}\right|^{2}\right) \Psi=-\left(1+\tilde{C}^{2}\right) \Psi$, where $\left\{e_{1}, e_{2}\right\}$ is an orthonormal frame on $T \tilde{\Sigma}$. Using the Gauss equation for $\tilde{\Phi}$, we obtain $\tilde{L}(\Psi)=-2 \tilde{C}^{2} \Psi+|\tilde{\sigma}|^{2} \Psi=$ $-2 \tilde{K} \Psi$. Hence,

$\tilde{Q}(\tilde{\varphi} \Psi)=-\int_{\tilde{\Sigma}} \tilde{\varphi} \tilde{\Delta} \tilde{\varphi} d \tilde{A}+\int_{\tilde{\Sigma}} 2 \tilde{\varphi}^{2} \tilde{K} d \tilde{A}-\int_{\tilde{\Sigma}}\left\langle\nabla_{\tilde{\nabla} \tilde{\varphi}^{2}}^{E} \Psi, \Psi\right\rangle d \tilde{A}=\int_{\tilde{\Sigma}}\left(|\tilde{\nabla} \tilde{\varphi}|^{2}+2 \tilde{\varphi}^{2} \tilde{K}\right) d \tilde{A}$

As $\tilde{K}$ and $\tilde{\varphi}$ are even with respect to $\tau$, together (4.4) and the above computation imply that, for any $\varphi \in C_{0}^{\infty}(\Sigma)$,

$$
0 \leq \tilde{Q}(\tilde{\varphi} \Psi)=\frac{1}{2} \int_{\Sigma}\left(|\nabla \varphi|^{2}+2 K \varphi^{2}\right) d A \leq \int_{\Sigma}\left(|\nabla \varphi|^{2}+K \varphi^{2}\right) d A .
$$

The above inequality means that the Schrödinger operator $\Delta-K$, on the complete and orientable Riemannian surface $\Sigma$, satisfies $\operatorname{Index}(\Delta-K)=0$.

From Theorem 4.2 , as $\Sigma$ is non compact, we have that either $\Sigma$ is conformally equivalent to $\mathbb{C}$, which is impossible because $\Sigma$ admits a connected two-fold covering, or $\Sigma$ is a flat cylinder. In this case (see Section 3), it is not difficult to check that $\Delta\left|\xi^{\top}\right|^{2}=4\left(1-\left|\xi^{\top}\right|^{2}\right)^{2}$. So $\left|\xi^{\top}\right|^{2}$ is a subharmonic function that satisfies $\left|\xi^{\top}\right|^{2} \leq 1$. As $\Sigma$ is complete and flat, the maximum principle implies that $\left|\xi^{\top}\right|^{2}$ is constant, and so $\left|\xi^{\top}\right|^{2}=1$. In particular $\Phi$ is a totally geodesic immersion. Hence $\Sigma$ must be a finite covering of the totally geodesic embedding of $\mathbb{R P}^{1} \times \mathbb{R}$ into $\mathbb{R} \mathbb{P}^{2} \times \mathbb{R}$, but, among such coverings, only $\mathbb{R} \mathbb{P}^{1} \times \mathbb{R}$ is stable.

The proof given for Theorem 4.10, with minor changes, allows to prove:

Theorem 4.12. Let $\Phi: \Sigma \rightarrow \mathbb{R P}^{2} \times \mathbb{S}^{1}(r)$ be a minimal immersion of an orientable complete surface. Then $\Phi$ is stable if and only if

1) $\Sigma=\mathbb{S}^{2}, \Phi(\Sigma)=\mathbb{R P}^{2} \times\{p\}, p \in \mathbb{S}^{1}(r)$, or

2) $\Phi$ is the totally geodesic immersion of $\mathbb{R P}^{1} \times \mathbb{R}$ into $\mathbb{R P}^{2} \times \mathbb{S}^{1}(r)$, or

3) $\Phi$ is the totally geodesic embedding of $\mathbb{R P} \mathbb{P}^{1} \times \mathbb{S}^{1}(r)$ into $\mathbb{R P}^{2} \times \mathbb{S}^{1}(r)$. 


\section{References}

[1] do Carmo, M., Peng, C. K.: Stable complete minimal surfaces in $\mathbb{R}^{3}$ are planes. Bull. Amer. Math. Soc. 1 (1979) 903-906.

[2] do Carmo, M., Ritoré, M. and Ros, A.: Compact minimal hypersurfaces with index one in the real projective space. Comment. Math. Helv. 75 (2000), no. 2, $247-254$.

[3] Farkas, H. M. And Kra, I.: Riemann surfaces. Graduate Texts in Mathematics 71, Springer-Verlag, New York-Berlin, 1980.

[4] Fischer-Colbrie, D.: On complete minimal surfaces with finite Morse index in three-manifolds. Invent. Math. 82 (1985), no. 1, 121-132.

[5] Fischer-Colbrie, D. And Schoen, R.: The structure of complete stable minimal surfaces in 3-manifolds of nonnegative scalar curvature. Comm. Pure Appli. Math. 33 (1980), no. 2, 199-211.

[6] López F. J. And Ros A.: Complete minimal surfaces with index one and stable constant mean curvature surfaces. Comment. Math. Helv. 64 (1989), no. 1, 34-43.

[7] Micallef M. J. and Wolfson J. G.: The second variation of area of minimal surfaces in four-manifolds. Math. Ann. 295 (1993), no. 2, 245-267.

[8] Montiel, S. And Urbano, F.: Second variation of superminimal surfaces into selfdual Einstein four-manifolds. Trans. Amer. Math. Soc. 349 (1997), no. 6, 2253-2269.

[9] Он, Y. G.: Second variation and stabilities of minimal Lagrangian submanifolds in Kähler manifolds. Invent. Math. 101 (1990), no. 2, 501-519.

[10] Ohnita, Y.: Stable minimal submanifolds in compact rank one symmetric spaces. Tôhoku Math. J. (2) 38 (1986), no. 2, 199-217.

[11] Pogorelov, A. V.: On the stability of minimal surfaces. Soviet Math. Dokl. 24 (1981), 274-276.

[12] Ros, A.: One-sided complete stable minimal surfaces. J. Differential Geom. 74 (2006), no. 1, 69-92.

[13] Ross, M.: Complete nonorientable minimal surfaces in $\mathbb{R}^{3}$. Comment. Math. Helv. 67 (1992), no. 1, 64-76.

[14] Simons, J.: Minimal varieties in riemannian manifolds. Ann. of Math. (2) 88 (1968), $62-105$.

[15] Torralbo, F. And Urbano, F.: On stable compact minimal submanifolds. To appear in Proc. Amer. Math. Soc. ArXiv: 1012.0679 [math.DG].

[16] Urbano, F.: Minimal surfaces with low index in the three-dimensional sphere. Proc. Amer. Math. Soc. 108 (1990), no. 4, 989-992.

Received June 16, 2011.

Francisco Urbano: Departamento de Geometría y Topología, Universidad de Granada, 18071 Granada, Spain.

E-mail: furbano@ugr.es

Research partially supported by MCyT-Feder research project MTM2007-61775, MEyC research project MTM2011-22547, and the Junta de Andalucía Grant P06-FQM-01642. 